Please cite as: K.L. Aplin and R.A. McPheat, An infra-red filter radiometer for atmospheric cluster-ion detection, Rev. Sci. Instrum., 79, 106107 (2008) doi:/10.1063/1.3002428

\title{
An infrared filter radiometer for atmospheric cluster-ion detection
}

K.L. Aplin* and R.A. McPheat

Space Science and Technology Department, Rutherford Appleton Laboratory, Chilton, Didcot, Oxon OX11 0QX UK

*corresponding author, email: k.1.aplin@,rl.ac.uk

\begin{abstract}
Motivated by the detection of infra-red absorption bands from atmospheric clusterions in laboratory experiments, a narrowband filter radiometer has been constructed for ion detection in the atmosphere. It uses a filter centred on $9.15 \mu \mathrm{m}$ wavelength with $5 \%$ bandwidth, protected by a diamond-like carbon coated germanium auxiliary filter, and fitted to a standard pyrradiometer. A sensitivity of $(29.3 \pm 0.1) \mu \mathrm{V} /\left(\mathrm{Wm}^{-2}\right)$ has been determined in a laboratory calibration using a blackbody source. Atmospheric experiments show that the filter radiometer, when used with a stable, low-noise amplifier, can respond to cluster-ion fluctuations. The filter radiometer's sensitivity to atmospheric ion changes is $(0.47 \pm 0.05)\left(\mathrm{mWm}^{-2}\right) /\left(\right.$ ions $\left.\mathrm{cm}^{-3}\right)$.
\end{abstract}

\section{Introduction and Motivation}

Cluster-ions are formed in the Earth's atmosphere, and other planetary atmospheres, by ionisation from cosmic rays and, near the continental surface, natural radioactivity ${ }^{1}$. They consist of an ion surrounded by hydrogen bonded ligands, which 
Please cite as: K.L. Aplin and R.A. McPheat, An infra-red filter radiometer for atmospheric cluster-ion detection, Rev. Sci. Instrum., 79, 106107 (2008) doi:/10.1063/1.3002428

are often, but not always, water ${ }^{2}$. Similar to neutral molecules and clusters, the stretching and bending modes of the hydrogen bonds cause infra-red (IR) absorption. Infra-red absorption by charged clusters was first identified by Carlon $^{3}$, and two absorption bands were subsequently accurately determined, centred at $9.15 \mu \mathrm{m}$ and 12.3 $\mu \mathrm{m}$, in artificial air at room temperature and pressure containing a corona source $^{4}$. In [4] a relative absorption signal of $\sim 3 \%$ was reported between $9.5-8.8 \mu \mathrm{m}$ by comparing ambient ion concentrations to those enhanced artificially with the corona source.

IR absorption bands from trace gases are commonly used for remote sensing; therefore identification of atmospheric IR absorption from cluster-ions permits remote sensing of charged species. As the ion column concentrations used in the spectroscopy cell in [4] $\sim 10^{13} \mathrm{~m}^{-2}$, and the atmospheric column ion concentration is $\sim 10^{14} \mathrm{~m}^{-2}$, it is possible in principle to detect atmospheric ion absorption. This approach would circumvent the practical difficulties of using sensitive electrical instrumentation for in situ atmospheric measurements ${ }^{5,6}$. Here we describe the design, construction, calibration and testing of a tuned narrowband filter radiometer, ruggedized for atmospheric deployment, which responds to atmospheric cluster ions in the $9.15 \mu \mathrm{m}$ band identified in [4].

\section{Filter Design and Characterisation}

Pyrradiometers are used for measuring broadband atmospheric longwave (IR) and solar radiation. The sensor is a blackened plate in thermal contact with a thermopile which responds to the radiation emitted or absorbed ${ }^{7}$. In normal operation, they 
Please cite as: K.L. Aplin and R.A. McPheat, An infra-red filter radiometer for atmospheric cluster-ion detection, Rev. Sci. Instrum., 79, 106107 (2008) doi:/10.1063/1.3002428

require measurement of the instrument body temperature so that the IR emission of the instrument itself can be determined and subtracted. The sensitivity of commercially available radiometers $\sim 100 \mu \mathrm{V} /\left(\mathrm{Wm}^{-2}\right)$, which, for typical broadband atmospheric downwelling longwave radiation $\sim 300 \mathrm{Wm}^{-2}$, gives a signal $\sim 30 \mathrm{mV}$. To detect the cluster ion absorption signal, a filter would be needed for the radiometer to pass the $9.15 \mu \mathrm{m}$ absorption band. A perfect filter with a Gaussian response centred on the peak absorption at $9.15 \mu \mathrm{m}$ would have a total transmissivity $\tau \sim 2 \%$ with respect to the black body spectrum over typical atmospheric surface temperatures. Typical filter radiometer signals in this band are expected to be $\sim 60 \mu \mathrm{V}$. This signal is above the noise level of available amplifiers $(5 \mu \mathrm{V})^{8}$.

Based on the sensitivity specification discussed above, and the additional requirements of durability and practicality, a filter head for a standard radiometer has been designed and constructed from anodized aluminium, Figure 1. The filter head was fitted onto a Swissteco ${ }^{9}$ pyrradiometer in place of the usual polythene dome. The filter head comprises a narrowband interference filter ${ }^{10}$ with $5 \%$ bandwidth, centred on $9.15 \pm 0.07 \mu \mathrm{m}$. An auxiliary filter was mounted vertically above the narrowband filter. It was constructed of germanium, with its outer face coated with diamond-likecarbon, and its inner face covered with high efficiency anti-reflection coating optimised for $8-12 \mu \mathrm{m}^{11}$. The purpose of the auxiliary filter was (1) to provide a longwavelength cutoff and (2) to provide robust environmental protection for the narrowband filter. Both filters were $30 \mathrm{~mm}$ diameter $\mathrm{x} 1 \mathrm{~mm}$ thick. The space inside the filter head (including between the two filters, see Figure 1) was purged with nitrogen to expel any residual water vapour, and sealed. The downwelling face of the pyrradiometer was covered and maintained at the instrument body temperature, which 
Please cite as: K.L. Aplin and R.A. McPheat, An infra-red filter radiometer for atmospheric cluster-ion detection, Rev. Sci. Instrum., 79, 106107 (2008) doi:/10.1063/1.3002428

was measured with a platinum resistance thermometer (PRT), in good thermal $\operatorname{contact}^{12}$.

The spectral characteristics of the narrowband and auxiliary filters were provided by their manufacturers ${ }^{10,11}$ and are shown in Figure 2a and b. (Manufacturer's data for the auxiliary filter for $\lambda>26 \mu \mathrm{m}$ was not available, so reference data was used for pure Ge for the long wavelength response ${ }^{13}$ ) The response of the filter radiometer can be calculated by multiplying the individual filter responses together, and was also measured using a Bruker 125 HR Fourier Transform Spectrometer, Figure 2c. The measured response (Figure 2c) was consistent with calculation. The transmissivity of the radiometer over the atmospheric longwave range can be found by integrating under Figure $2 \mathrm{c}$ and is $5.8 \%$ of the black body spectrum for typical atmospheric temperatures. The temperature sensitivity of the transmissivity due to the change in the black body peak is $<0.01 \%$ for -10 to $30^{\circ} \mathrm{C}$ and can be neglected.

\section{Laboratory Calibration}

The filter radiometer was calibrated using an Isotech Hyperion R black body source, with the black body temperature maintained to $\pm 0.1 \mathrm{~K}$. The filter radiometer's sensing head was placed close to the source so that the black body filled its entire field of view. The temperature of the black body source was varied from -10 to $30^{\circ} \mathrm{C}$ and the instrument body temperature was allowed to stabilise before manual measurements of the black body temperature, radiometer signal and PRT resistance were made. A precision $\times 500$ gain stage was used to amplify the signal ${ }^{8}$. To allow for a wider variation in instrument body temperature, to mimic atmospheric operation, a second 
Please cite as: K.L. Aplin and R.A. McPheat, An infra-red filter radiometer for atmospheric cluster-ion detection, Rev. Sci. Instrum., 79, 106107 (2008) doi:/10.1063/1.3002428

calibration experiment was carried out. In this experiment the black body source was kept at a constant $10^{\circ} \mathrm{C}$ and the instrument body temperature allowed to vary. The body temperature was sampled every 30 s with a Keithley 2701 four-wire resistance meter, and logged to a PC. The radiometer thermopile voltage was sampled every $20 \mathrm{~s}$ using a Campbell CR3000X data logger, and five minute averages were calculated for both instruments.

Assuming no absorption of radiation in the $\sim 1 \mathrm{~cm}$ air gap between the blackbody at a constant temperature $T_{b}$ and the radiometer body at temperature $T_{r}$, the output signal in volts $V_{\mathrm{s}}$ is given by

$$
V_{s}=\frac{k \tau \sigma}{G}\left(T_{b}^{4}-T_{r}^{4}\right)(1)
$$

where $\sigma$ is Stefan's constant, $k$ the radiometer sensitivity, $\tau$ the transmissivity and $G$ the amplifier gain. Plotting the measured signal $\left(G V_{s}\right)$ against $\sigma T_{r}^{4}$ will give an intercept of $k \tau \sigma T_{b}^{4}$ and a gradient of - $\tau \mathrm{k}$. In the experiment, the incoming blackbody radiation $\sigma T_{b}^{4}$ is constant at $364 \pm 15 \mathrm{Wm}^{-2}$, so if $\tau$ is known, $k$ can be found. The sensitivity was determined as $k=(29.3 \pm 0.1) \mu \mathrm{V} /\left(\mathrm{Wm}^{-2}\right)$.

\section{Atmospheric Test}

In the atmosphere, the IR radiation is modulated by the absorption of cluster-ions in the column above the instrument. The atmospheric absorption in the filter pass band $\left(L_{\lambda}\right)$ is determined by the difference between the reference signal $V_{S}(1)$, the radiation balance between the emission of the radiometer body and the downwelling longwave radiation (where the atmosphere is assumed to emit as a black body), and the measured signal $V_{r}$. 
Please cite as: K.L. Aplin and R.A. McPheat, An infra-red filter radiometer for atmospheric cluster-ion detection, Rev. Sci. Instrum., 79, 106107 (2008) doi:/10.1063/1.3002428

$$
L_{\lambda}=\frac{\left(V_{r}-V_{s}\right)}{k G} \tau(2)
$$

In an atmospheric test, the filter radiometer was run at a meteorological site with atmospheric electrical sensors (described in more detail in [1]). The atmospheric conductivity was determined by Ohm's law from measurements of the atmospheric Potential Gradient $(\mathrm{PG})^{14}$ and conduction current density ${ }^{15}$, the drift current of atmospheric ions flowing in the global electric circuit ${ }^{1}$. The air ion concentration can be determined from the atmospheric conductivity if a constant ion mobility of 1.7 $\mathrm{cm}^{2} \mathrm{~V}^{-2} \mathrm{~s}^{-1}$ is assumed ${ }^{1}$. Errors in the air ion concentration from possible ion mobility variations $^{6}$ are $\sim 7.5 \%$. Data from a foggy, meteorologically quiescent night in November 2007 was selected to exclude any effects of variations in shortwave solar radiation, wind speed and water vapour. Simultaneous ion concentration and filter radiometer measurements can be seen in Figure 3, showing that the instrument sensitivity to atmospheric ions is $0.47 \pm 0.05\left(\mathrm{mWm}^{-2}\right) /\left(\right.$ ions $\left.\mathrm{cm}^{-3}\right)$. The response in fog indicates that the instrument can respond to electrical effects in saturated conditions, suggesting that remote sensing of ions through or in cloud may be possible.

\section{Acknowledgements}

B.J. Landowski (Rutherford Appleton Laboratory - RAL) designed the filter head and Figure 1 was produced by M.S. Whalley (RAL). Technical support from W. Robins (RAL) and A.G. Lomas (University of Reading) is acknowledged. K. A. Nicoll, R.G. Harrison (University of Reading), M. Clapp and D. Weidmann (RAL) assisted with the measurements. This project was partially funded by the Science and Technology Facilities Council and the Natural Environment Research Council. 
Please cite as: K.L. Aplin and R.A. McPheat, An infra-red filter radiometer for atmospheric cluster-ion detection, Rev. Sci. Instrum., 79, 106107 (2008) doi:/10.1063/1.3002428

\section{Figure Captions}

Figure 1 Filter radiometer head, constructed of anodized aluminium, showing inlets for purging, and the two filters. Filter dimensions are $30 \times 1 \mathrm{~mm}$. (a) three dimensional rendering (b) cross section.

Figure 2 Filter radiometer spectral response (a) Upper germanium auxiliary filter, data provided by Crystran Ltd. for $0<\lambda<26 \mu \mathrm{m}$ and from reference data for $\lambda>26 \mu \mathrm{m}^{13}$ (b) Narrowband $9.15 \mu \mathrm{m}$ filter, data provided by Northumbria Optical Coatings Ltd. (c) Measured response of both filters, which can also be calculated by multiplying (a) and (b).

Figure 3 Filter radiometer response to changes in ion concentration for midnight-6am on 5th November 2007 (386 points), in foggy conditions. The gradient is $0.47 \pm 0.05$ $\left(\mathrm{mWm}^{-2}\right) /\left(\right.$ ions $\left.\mathrm{cm}^{-3}\right)$

\section{References}

${ }^{1}$ K.L. Aplin, Space Sci. Rev., in press, doi:10.1007/s11214-008-9397-1 (2008)

${ }^{2}$ R.G. Harrison and K.S. Carslaw, Rev. Geophys. 41 doi:10.1029/2002RG000114 (2003)

${ }^{3}$ H.R. Carlon and C.S. Harden, Applied Optics 19 (11), 1776-1786 (1980)

${ }^{4}$ K.L. Aplin and R.A. McPheat, J. Atmos. Sol-Terr Phys., 67(8-9), 775-783. doi:10.1016/j.jastp.2005.01.007 (2005)

${ }^{5}$ K. A. Nicoll and R.G. Harrison, Rev. Sci. Instrum, 79, 084502 doi:

$10.1063 / 1.2964927(2008)$

${ }^{6}$ R.G. Harrison and K.L. Aplin, Atmos. Res. 85, 199-208

doi:10.1016/j.atmosres.2006.12.006 (2007) 
Please cite as: K.L. Aplin and R.A. McPheat, An infra-red filter radiometer for atmospheric cluster-ion detection, Rev. Sci. Instrum., 79, 106107 (2008) doi:/10.1063/1.3002428

${ }^{7}$ W. D. Sellers, Physical climatology, The University of Chicago Press (1965)

${ }^{8}$ R.G. Harrison and J.R. Knight, Rev. Sci. Instrum. 77, 116105

doi:10.1063/1.2370752 (2006)

${ }^{9}$ Swissteco Instruments, CH-9463 Oberriet, Switzerland

${ }^{10}$ Northumbria Optical Coatings, Ltd. 10, Boldon Business Park, Burford Way, Boldon, Tyne and Wear, NE35 9PZ, UK (www.noc-ltd.com).

${ }^{11}$ Crystran Ltd., 1 Broom Road Business Park, Poole, Dorset, BH12 4PA, UK (www.crystran.co.uk).

${ }^{12}$ R.G. Harrison and G.W. Rogers, Rev. Sci. Instrum., 77, 116112, doi: $10.1063 / 1.2400013(2006)$

${ }^{13}$ J.S. Browder, S.S. Ballard and P. Klocek, Physical property comparisons of crystalline infrared optical materials In: P. Klocek (ed)., Handbook of Infrared Optical Materials, Marcel Dekker Inc., New York (1991)

${ }^{14}$ A.J. Bennett and R.G. Harrison, Rev. Sci. Instrum. 77, 016103 (2006)

${ }^{15}$ A.J. Bennett and R.G. Harrison, J. Atmos. Sol.-Terr. Phys., 70, 1373- 1381 doi:10.1016/j.jastp.2008.04.014 (2008) 


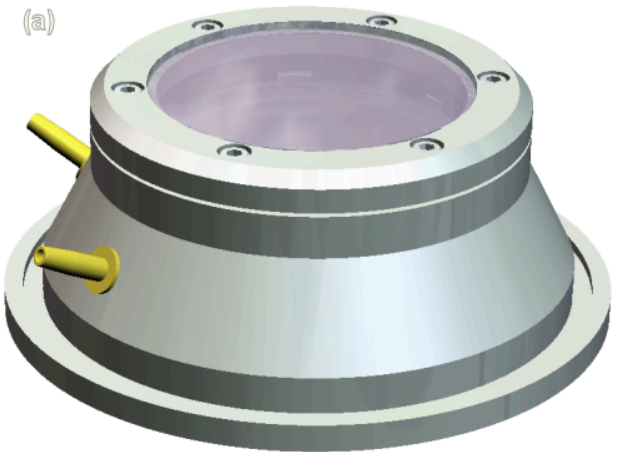

(b))

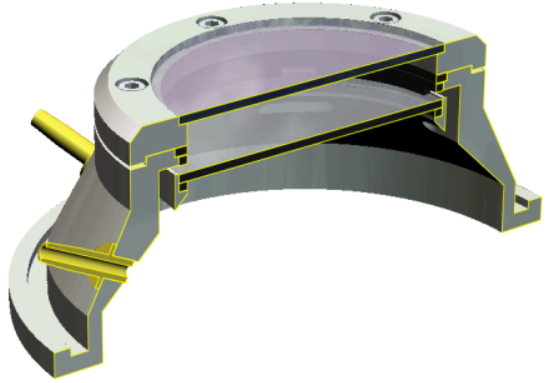


(a) Auxiliary filter

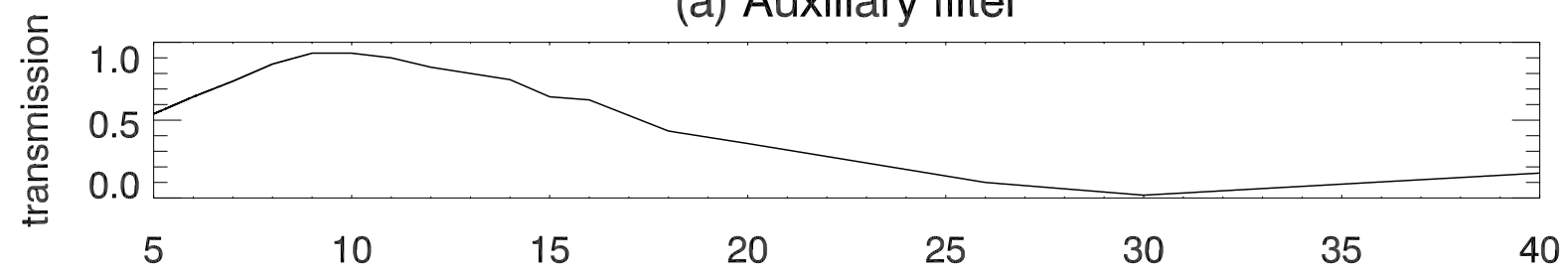

(b) Narrowband filter

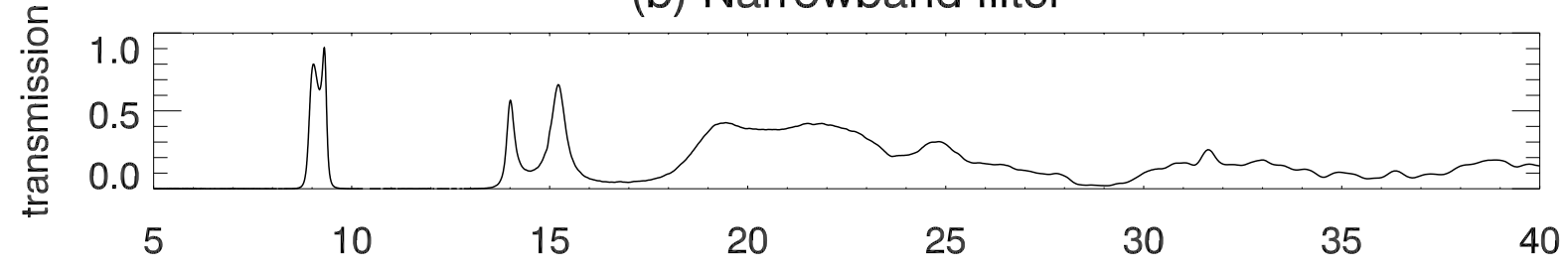

(c) Auxiliary plus narrowband filters

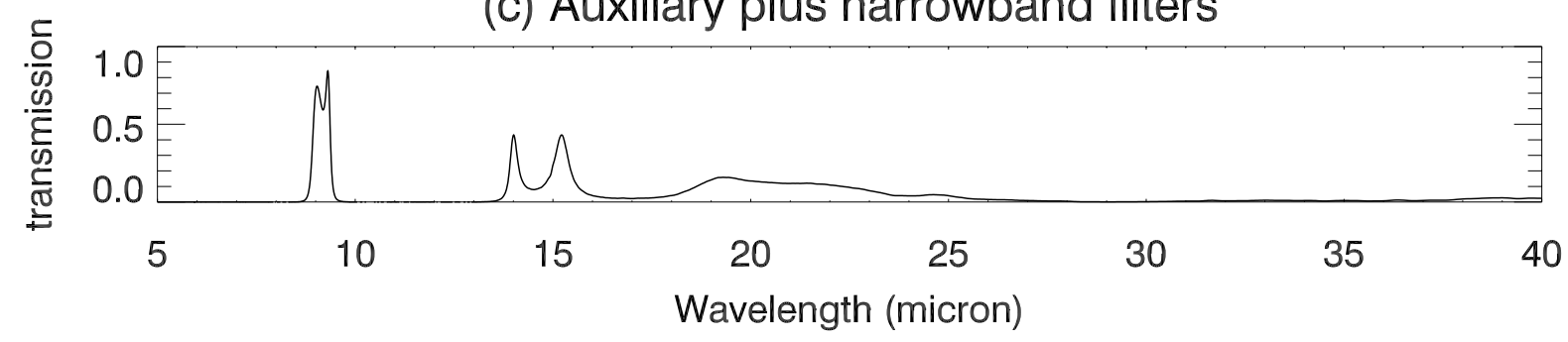


radiometer change $(\mathrm{mW} / \mathrm{m} 2)$

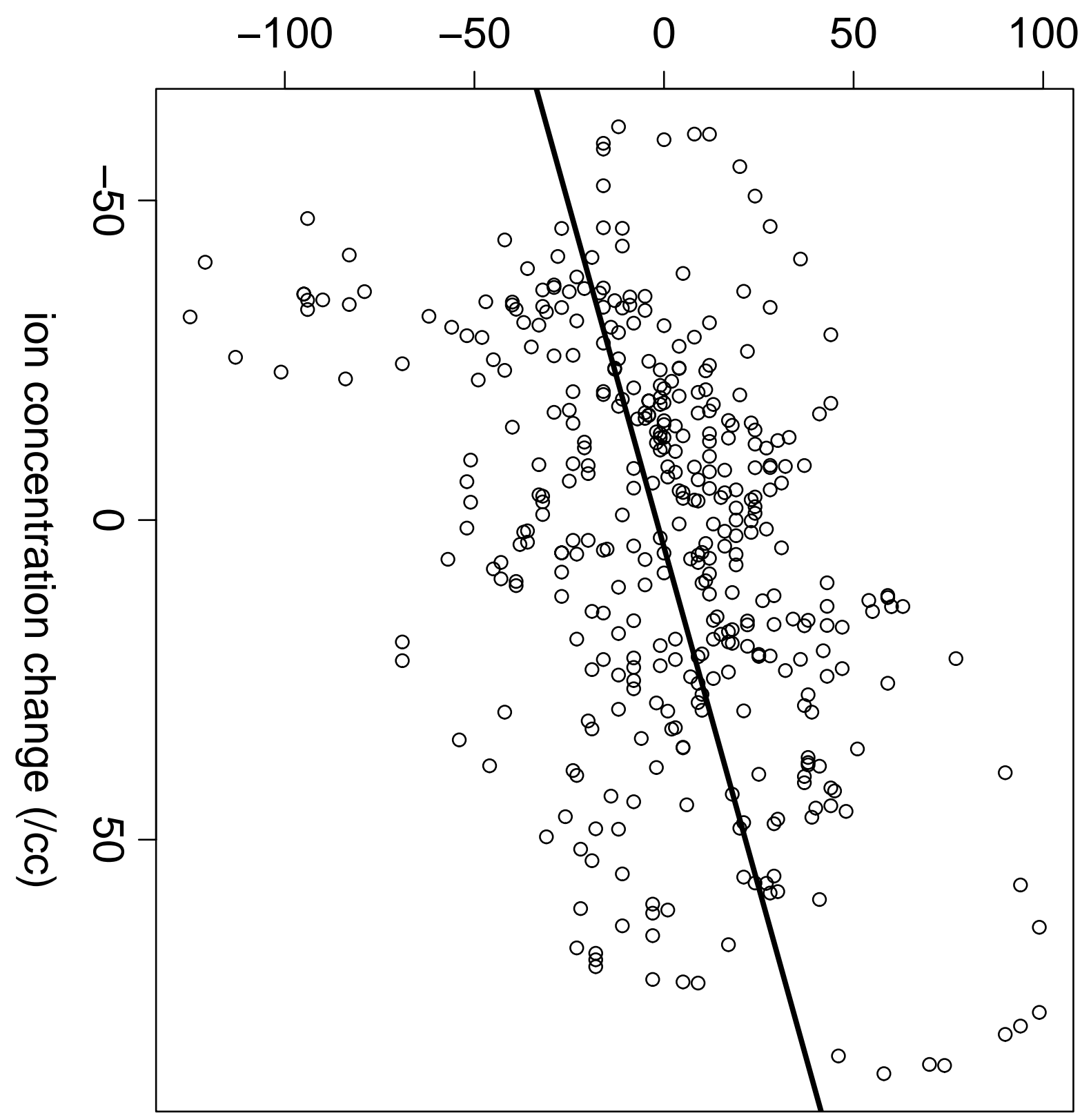

